\title{
Les peuplements d'éphémèroptères de plécoptères et de trichoptères des ruisseaux acides et non acides du massif vosgien : première approche Ephemeroptera, plecoptera, trichoptera communities of acidic and non acidic streams in the vosges mountains (northeastern France): a preliminary study
}

\author{
F. Guerold,, D. Vein et G. Jacquemin
}

Volume 4, numéro 3, 1991

URI : https://id.erudit.org/iderudit/705101ar

DOI : https://doi.org/10.7202/705101ar

Aller au sommaire du numéro

\section{Éditeur(s)}

Université du Québec - INRS-Eau, Terre et Environnement (INRS-ETE)

\section{ISSN}

0992-7158 (imprimé)

1718-8598 (numérique)

\section{Découvrir la revue}

Citer cet article

Guerold,, F., Vein, D. \& Jacquemin, G. (1991). Les peuplements

d'éphémèroptères de plécoptères et de trichoptères des ruisseaux acides et non acides du massif vosgien : première approche. Revue des sciences de l'eau / Journal of Water Science, 4(3), 299-314. https://doi.org/10.7202/705101ar

\section{Résumé de l'article}

Les peuplements d'Ephémèroptères, de Plécoptères et de Trichoptères de quatorze cours d'eau du massif vosgien granitique, ont été échantillonnés de mars 1988 à mars 1990. Les stations, au nombre de seize, ont été choisies en amont de toute agglomération et en dehors de zones d'activité agricole. Les mesures de $\mathrm{pH}$, conductivité et aluminium total, ont établi que sept ruisseaux sont acides ( $\mathrm{pH}$ moyens : 4,67-5,62), très faiblement minéralisés (conductivités moyennes : 17-21,5 $\mu \mathrm{S} / \mathrm{cm}$ ) et présentent des concentrations moyennes en aluminium total, comprises entre 221 et $387 \mu \mathrm{g} / \mathrm{l}$.

Les sept autres cours d'eau sont faiblement acides à neutres ( $\mathrm{pH}$ moyens : 6,80-6,98), peu minéralisés (conductivité moyenne : $36-90 \mu \mathrm{S} / \mathrm{cm}$ ) et montrent des concentrations moyennes eut aluminium variant de 41 à $78 \mu \mathrm{g} / \mathrm{l}$.

Seules 29 espèces ont été récoltées dans les ruisseaux acides alors que 93 l'ont été dans les cours d'eau non acides.

Les Ephémèroptères se révèlent les plus sensibles aux conditions acides et disparaissent totalement. Parmi les Trichoptères, seuls les Polycentropodidae, les Rhyacophilidae et les Limnephilidae sont encore présents dans les ruisseaux acides. Alors que les Plécoptères Filipalpes sont bien représentés, certaines espèces étant même très abondantes, les Plécoptères Sétipalpes ne sont plus récoltés dans les cours d'eau acidifiés, à l'exception de Siphonoperla torrentium. 


\title{
Les peuplements d'éphémèroptères de plécoptères et de trichoptères des ruisseaux acides et non acides du massif vosgien : première approche ${ }^{*}$
}

\author{
Ephemeroptera, plecoptera, trichoptera communities \\ of acidic and non acidic streams \\ in the vosges mountains (northeastern France): \\ a preliminary study
}

\section{F. GUEROLD1', D. VEIN², G. JACQUEMIN³}

Reçu le 26 juillet 1990, accepté pour publication le 28 janvier $1991^{* *}$.

RÉSUMÉ

Les peuplements d'Ephémèroptères, de Plécoptères at de Trichoptères de quatore cours d'eau du massil vosgien granitique, ont été échantillonnés de mars 1988 à mars 1990. Les stations, au nombre de seize, ont été choisles en amont de toute agglomération et en dehors de zones d'activité agricole.

Les mesures de $\mathrm{pH}$, conductivité et aluminium total, ont établi que sept ruisseaux sont acides ( $\mathrm{pH}$ moyens : 4,67-5,62), très faiblement minéralisés (conductivités moyennes : $17-21,5 \mu \mathrm{S} / \mathrm{cm}$ ) et présentent des concentrations moyennes en aluminium total, comprises entre 221 et $387 \mu \mathrm{g} / \mathrm{l}$.

Les sept autres cours d'eau sont faiblement acides à neutres (pH moyens : $6,80-6,98$ ), peu minéralisés (conductivité moyenne : $36-90 \mu \mathrm{S} / \mathrm{cm}$ ) et montrent des concentrations moyennes en aluminium variant de 41 à $78 \mu 0 /$.

Seules 29 espèces ont été récoltées dans les ruisseaux acides alors que 93 I'ont été dans les cours d'eau non acides.

Les Ephémèroptères se révèlent les pius sensibles aux conditions acides et disparaissent totalement. Parmi les Trichoptères, seuls les Polycentropodidae, les Rhyacophilidae et les Limnephilidae sont encore présents dans les ruisseaux acides. Alors que les Plécoptères Filipalpes sont bien représentés, certaines espèces étant même très abondantes, les Plécoptères Sétipalpes ne sont plus récoltés dans les cours d'eau acidifiés, à l'exception de Siphonoperla torrentium

1. Université de Metz, Faculté des Sciences, Laboratoire d'Ecologie, BP 4116, 57040 Metz, cedex 01.

2. Ecole Nationale Supérieure o'Agronomie et des Industries Alimentaires, BP 172, 54505 Vandoeuvre-les-Nancy, cedex.

3. Université de Nancy I, Faculté des Sciences, Laboratoire de Biologie des Insectes, BP 239, 54506 Vandoeuvreles-Nancy cedex.

* Communication présentée au 34e Congrès de l'Association Française de Limnologie, Metz-Nancy, $29-31$ mai 1990.

* Les commentaires seront reçus jusqu'au 30 mars 1992. 
Mots clés : Eaux courantes, acidification, éphémèroptères, trichoptères, plécoptères, bio-indicateurs, massit vosgien.

Acidification of freshwaters has become a serious problem in certain parts of the Vosges Mountains (Northeastern France). Aquatic organisms at all major levels are affected by decreased $\mathrm{pH}$. As the Ephemeroptera, Plecoptera and Trichoptera are a significant par of the macrobenthos in mountain streams, the objective of this preliminary study was to determine how the physico-chemical environment in acidilied running waters affects the qualitative composition of maytlies, stoneflies and caddis-filies communities compared with non acidified streams and to evaluate the reliability and possible use of the taxonomic groups as indicators of the extent of acidification.

A biological survey of fourteen streams was conducted in the Vosges mountains from March 1988 to March 1990. The areas investigated lie on granitic bed-rock and soils in the process of podzolisation. The sampling sites (sixteen), at altitudes of 600 to 1020 meters a.s.I. were located above built-in and agricultural areas thus avoiding organic pollution. The streams drain forested catchments regarted as sensitive to acid inputs and affected by forest decline. Silver fir (Ables alba), Norway spruce (Pecea abies) and European beech (Fagus syivalica) are dominant.

Larvae and pupae of Ephemeroptera, Plecoptera and Trichoptera were collected by hand picking and Surber sampler (mesh aperture $350 \mu \mathrm{m}$ ). Adults were collected with an insect net by beating bushes and trees or sweeping low herbage near streams. A long-handled net was required for high-flying Ephemeroptera and Trichoptera. Organisms were preserved in $5 \%$ formalin or $70 \%$ ethanol.

The $\mathrm{pH}$ was measured in the field using a specific glass electrode for low ionic solutions, compensated for temperature. Water samples were aciditied for total aluminium analysis. Determination of aluminium was performed using an atomic absorption spectrophotometer equipped with a graphite furnace. Conductivity was measured in the laboratory at $20^{\circ} \mathrm{C}$.

Determination of $\mathrm{pH}$ revealed that acidification occurred at seven sites (sampling sites $\mathrm{a}^{\circ} 8$ to 16). Six were strongly acidic (mean $\mathrm{pH}: 4.7-5.2$ ) and one moderately acidic (mean pH : 5,6). In such streams mean total aluminium ranged from $221 \mu \mathrm{g} / \mathrm{h}$ to $387 \mu \mathrm{g} / \mathrm{h}$ and mean conductivity from $17,0 \mu \mathrm{S} / \mathrm{cm}$ to $21.5 \mu \mathrm{S} / \mathrm{cm}$. Minimum $\mathrm{pH}$ and maximum aluminium values were recorded during snowmelt or rain event. In all these streams the fish population (Salmo trutta fario) has completely disappeared.

Others streams (sampling sites $n^{\circ} 1$ to 7 ) went from slightly acidic to neutral with a range $\mathrm{pH}$ between 6.8-7.0 and were characterized by average total aluminium varying from $41 \mu \mathrm{g} / \mathrm{to} 78 \mu \mathrm{g} / \mathrm{h}$ and average conductivity from $36 \mu \mathrm{S} / \mathrm{cm}$ of $90 \mu \mathrm{S} / \mathrm{cm}$. These streams have been considered as reference for non acidified running waters.

The number of species occurring in acidified streams contrasted markedly with those of the non acidified streams. Specific richness of Ephemeroptera, Plecoptera and Trichoptera are reduced in acidic waters. Only twenty-nine species were found in acidic streams, whereas a total of ninety-three species were collected in non acidic streams.

\section{Ephemeroptera}

In the reference streams, the Ephemeroptera fauna was rich and diverse with twenty taxa. Some species, for example Epeorus sylvicola, Baelis alpinus and 
B. rhodani were very common and widespread. In contrast, in acidic water only one species, Baetis vernus, was actualy present ( 3 larvae).

\section{Trichoptera}

Thirty-eight species of caddis flies were found in non acidic streams. Hydropsychidae (hydropsyche sp) Philopotamidae (Philopotamus ludificalus, $P$. montanus, Wormaldia sp) and Glossosomatidae (Glossosoma conformis, Agapefus sp) were the organisms most commonly present.

In acidic streams, eleven species were found. Rhyacopyhilidae (Rhyacophila obliterata, $\boldsymbol{R}$. potamoides, $\boldsymbol{A}$. praemorsa), Polycentropodidae (Plectrocnemia $s p, P$. conspersa) and Limnephilidae (Drusus annulatus, D. discojor, Caetopterygopsis maclachlani, Chaetopteryx villosa, Anitella sp) were among the most abundant taxa.

\section{Plecoptera}

In acidic streams, taxonomic richness (17 species) was about $50 \%$ lower than those obtained in the non acidic streams. The common species Perla marginata, Perlodes microcephala and /soperla oxylepis were not listed when $\mathrm{pH}$ was below 5.6. At the opposit, some species were dominant and very abundant. For example, Brachyptera seficornis, was dominant in April, Leuctra nigra in May, Siphonopenta torrentium in June and Leuctra cingulata in July.

The sensitivity of organisms towards to acidification appeared to be different among these groups of aquatic insects. Three types of responses can be disInguished in relation with $\mathrm{pH}$ and/or associated factors :

- High sensibility of Ephemeroptera, which have completely disappeared

- High sensitivity of most Trichoptera families except the following : Polycentropodidae, Rhyacophilidae and Limnephilidae.

- Tolerance of Plecoptera tilipalpia which were sometimes very abundant, but disappearance of Plecoptera Setipalpia except for Siphonoperla torrentium.

This analysis showed that many species of mayflies, stoneflies and caddis flies do nol tolerate low $\mathrm{pH}$ (and/or) associated with low conductivity and elevated aluminium concentrations. Because the biological response is the result of present and post environmental situations, we suggest the use of these taxa as indicators of acidification in running waters, particularly when a restricted number of physico-chemical measurements is insufficient to appreciate the acidification level, as during low flow periods or during periodes of no acid stress.

Nevertheless, to be considered as a useful indicator of pollution in general and acidification in particular, a taxa (species, genera or familly) should be widespread, relatlvely abundant, present all the year round, very sensitive to the studied factor and easy or relatively easy to identify.

For example, in unpoltuted streams in the Vosges Mountains, Epeorus sylvcola, Periodes microcephala, Hydropsyche sp. Philopotamus sp seem to fit these criteria.

However, in evaluating aquatic organisms as indicators of pollutional conditions, great caution is necessary because knowledge of the ecological requirements of the species is essential, several ecologic conditions other than the presence of a pollutant may limit the distribution of certain species. For this reason, the benthic macrolauna of a more signiticant number of sites of different chemical and physical characteristics should be collected.

Key-words : Running waters, acidification, Ephemeroptera, Plecoptera, Trichoptera, bio-indicators, Vosges Mountains. 


\section{1 - INTRODUCTION}

L'acidification des eaux de surface en Amérique du Nord et en Europe, est aujourd'hui reconnue comme étant l'un des problèmes d'environnement les plus importants.

Les retombées atmosphériques acides, conséquences des émissions anthropogéniques dans l'atmosphère de $\mathrm{SO}_{2}$ et $\mathrm{No}_{x}$ induisent des altérations des composantes chimiques et biologiques des écosystèmes terrestres (PITELKA et RAYNAL, 1989 ; REHFUESS, 1989 ; SCOTT, 1989) et aquatiques (LEIVESTAD et MUNIZ, 1976 ; HENRIKSEN, 1979).

En France, dans le massif vosgien (nord-est), les premières données sur des précipitations datent de 1973 (BOURRIE, 1976). Depuis lors, des études ont montré que certains événements neigeux peuvent s'accompagner de valeurs extrêmes de $\mathrm{pH}$ de 3,05 (COLIN et al., 1989). La truite (Salmo trutta fario) a disparu de nombreux cours d'eau drainant des bassins versants vulnérables aux dépôts atmosphériques acides, du fait de la nature du substratum géologique, des sols et de la végétation.

Dans de tels cours d'eau les peuplements de macro-invertébrés benthiques montrent d'importantes modifications de leur composition, qui se traduisent notament par la disparition de nombreuses espèces (GUEROLD et PIHAN, 1989). Les macro-invertébrés, depuis longtemps utilisés comme bioindicateurs de pollution (GAUFIN et TARZWELL, 1982 ; WOODIWISS, 1964 ; BRINKURST, 1966 ; CAIRNS, 1974 ; VERNEAUX, 1984), reflètent la situation présente mais aussi passée d'un cours d'eau. Il apparaissent donc comme un matériel de choix pour appréhender à un moment donné l'état d'un écosystème lotique subissant une perturbation. Par ailleurs, il semble réalisable d'élaborer une méthode d'évaluation de l'état d'acidification des cours d'eau, basée sur l'utilisation des macro-invertébrés benthiques (WRIGHT et al., 1984 ; NORWEGIAN INSTITUTE FOR WATER RESEARCH, 1987).

C'est pourquoi nous nous sommes intéressés à trois groupes d'invertébrés caractéristiques des ruisseaux de montagne (Ephémèroptères, Plécoptères, Trichoptères). Le but de cette étude préliminaire était de mettre en évidence les différences qualitatives apparaissant au sein de chacun de ces trois groupes faunistiques, entre cours d'eau acidifiés et non acidifiés et de voir si leur utilisation en tant que bio-indicateur d'acidification était envisageable.

\section{2 - SITES D'ÉTUDE}

Quatorze cours d'eau du massif vosgien granitique ont été étudiés (fig. 1 et tableau 1). Huit d'entre eux (stations $n^{\circ} 7$ à 16) sont localisés sur un même bassin versant (bassin versant du Rouge-Rupt). Les six autres cours d'eau (stations $n^{\circ} 1$ à 6 ) sont situés dans des localités voisines. 
Tableau 1 Situation et codage des stations de prélèvement.

Table 1 Location and coding of sampling points.

\begin{tabular}{|c|c|c|c|c|}
\hline Cours d'eau & $\mathbf{N}^{\circ}$ & $\begin{array}{l}\text { Altitude } \\
\mathrm{M}\end{array}$ & Référence I.G.N.1 & $\begin{array}{l}\text { Coordonnées } \\
\text { Lattitude Nord \& Longitude Est }\end{array}$ \\
\hline Rau Saint Nicolas & 1 & 6050 & La Bresse 3619 Ouest & $47^{\circ} 55^{\prime} 10^{\prime \prime} \mathrm{N} \& 6^{\circ} 57^{\prime \prime} 21^{\prime \prime} \mathrm{E}$ \\
\hline Thur & 2 & 625 & La Bresse 3619 Ouest & $47^{\circ} 59^{\prime} 10^{\prime \prime} N \& 6^{\circ} 58^{\prime} 3^{\prime \prime} E$ \\
\hline Vologne & 3 & 810 & La Bresse 3619 Ouest & $48^{\circ} 3^{\prime} 18^{\prime \prime} \quad N \& 6^{\circ} 59^{\prime \prime} 3^{\prime \prime} E$ \\
\hline Meurthe & 4 & 825 & Gérandmer 3618 Est & $48^{\circ} 5^{\prime} 22^{\prime \prime} \quad \mathrm{N} \& 7^{\circ} 1^{\prime} 18^{\prime \prime} \mathrm{E}$ \\
\hline Bouchot & 5 & 730 & La Bresse 3619 ouest & $48^{\circ} 234^{\prime \prime} \quad \mathrm{N} \& 6^{\circ} 51^{\circ} 47^{\prime \prime} \mathrm{E}$ \\
\hline Rau des Bans & 6 & 630 & Gérardmer 3618 Ouest & $48^{\circ} 9^{\prime} 20^{\prime \prime} \quad N \& 66^{\circ} 52^{\prime} 36^{\prime \prime} E$ \\
\hline Goutte du Tihay & 7 & 660 & La Bresse 3619 Ouest & $47^{\circ} 59^{\prime} 8^{\prime \prime} \quad N \& 6^{\circ} 53^{\prime} 54^{\prime \prime} \mathrm{E}$ \\
\hline Rau des Echarges & 8 & 750 & La Bresse 3619 Ouest & $47^{\circ} 58^{\prime} 24^{\prime \prime} N \& 6^{\circ} 53^{\prime} 56^{\prime \prime} \mathrm{E}$ \\
\hline Rau du Grand Ventron & 9 & 810 & La Bresse 3619 Ouest & $47^{\circ} 58^{\prime} 42^{\prime \prime} N \& 6^{\circ} 54^{\circ} 41^{\prime \prime} \mathrm{E}$ \\
\hline Rouge-Rupt $673 \mathrm{~m}$ & 10 & 673 & La Bresse 3619 Ouest & $47^{\circ} 58^{\prime} 32^{\prime \prime} N \& 6^{\circ} 53^{\prime} 25^{\prime \prime} E$ \\
\hline Goutte des Blancs Murgers & 11 & 970 & La Bresse 3619 Ouest & $47^{\circ} 58^{\prime} 58^{\prime \prime}$ N \& 6 6 $55^{\prime} 34^{\prime \prime} \mathrm{E}$ \\
\hline Wassongoutte & 12 & 700 & La Bresse 3619 Ouest & $47^{\circ} 58^{\prime} 24^{\prime \prime} \mathrm{N} \& 6^{\circ} 53^{\prime} 4^{\prime \prime} \mathrm{E}$ \\
\hline Goutte de la Grande Basse & 13 & 840 & La Bresse 3619 Ouest & $47^{\circ} 58^{\prime} 55^{\prime \prime} N \& 6^{\circ} 55^{\prime} 23^{\prime \prime} \mathrm{E}$ \\
\hline Rouge-Rupt 845 m & 14 & 845 & La Bresse 3619 Ouest & $47^{\circ} 58^{\circ} 52^{\prime \prime} N \& 6^{\circ} 55^{\prime 2} 28^{\prime \prime} \mathrm{E}$ \\
\hline Goutte du Pourti Faing & 15 & 1010 & La Bresse 3619 Ouest & $47^{\circ} 58^{\circ} 57^{\prime \prime} \mathrm{N} \& 6^{\circ} 55^{\circ} 18^{\prime \prime} \mathrm{E}$ \\
\hline Rouge-Rupt $1020 \mathrm{~m}$ & 16 & 1020 & La Bresse 3619 Ouest & $47^{\circ} 59^{\circ} 54^{\prime \prime} \mathrm{N} \& 6^{\circ} 55^{\prime} 18^{\prime \prime} \mathrm{E}$ \\
\hline
\end{tabular}

Institut Géographique National carte au 1 : 25000 .

Chaque station se trouve en amont de toute agglomération et placée dans des secteurs non perturbés par l'activité agricole, exception faite de la station 1 qui se situe en aval d'un hameau.

Les stations 4,5 et 7 sont situées dans des zones non forestières. Les stations $3,6,8,9,10,11,12,13,14,15$ et 16 sont situées sous couvert forestier (conifères dominants). Les stations 1 et 2 sont situées sous couvert forestier (feuillus dominants).

\section{3 - MÉTHODOLOGIE}

\subsection{Physico-chimie}

A chaque station le $\mathrm{pH}$ est mesuré à laide d'un pH mètre portatif équipé d'une électrode spécifique pour les solutions faiblement ioniques. Des échantillons d'eau ont également été prélevés à plusieurs reprises afin de déterminer les concentrations en aluminium total. Pour cela l'eau est placée dans des bidons $(1 \mathrm{l})$ en polyéthylène et gardée au frais et à l'obscurité. Le pH est contrôlé au laboratoire et la conductivité mesurée à $20^{\circ}$. Après acidification des échantillons, l'aluminium est dosé dans les 48 heures par spectrophotométrie d'absorption atomique au four graphite. Pour chaque cours d'eau, au moins une campagne de mesure a été effectuée en période de fonte des neiges, événement hydrométéorologique reconnu critique pour l'environnement (ODEN, 1976 ; SCHOFIELD, 1976 ; VAN COILLIE et al., 1984). 

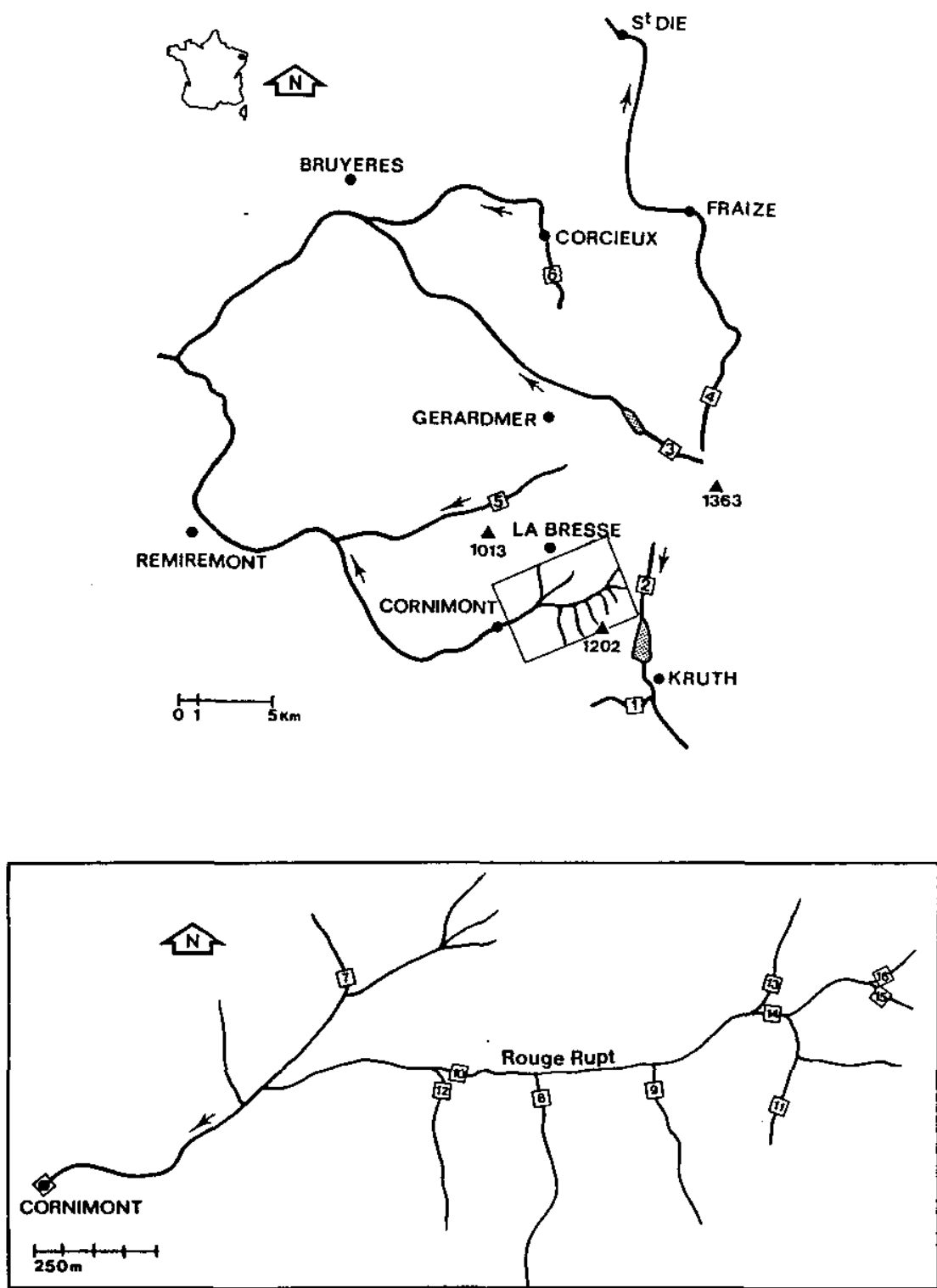

Figure 1 Localisation des sites d'étude et de stations de prélèvement. Map showing location of study area and sampling points. 


\subsection{Macro-invertébrés benthiques}

La macrofaune benthique a été échantillonnée mensuellement en 1988, 1989 et jusqu'en mars 1990 . Des prélèvements $\left(0,08 \mathrm{~m}^{2}\right)$ effectués en fonction de la diversité de couples courant /substrat, ont été réalisés à l'aide d'un filet Surber de $350 \mu \mathrm{m}$ de vide de maille. Les récoltes de larves ont été complétées par une prospection manuelle aussi exhaustive que possible. Les imagos d'Ephémèroptères, Plécoptères, et Trichoptères ont été récoltés à l'aide de filet d'entomologiste, de filet fauchoir et de parapluie japonais, ces deux dernières techniques étant surtout efficaces pour collecter les adultes de Plécoptères.

Les imagos et larves récoltés par chasse à vue sont conservés dans l'alcool à $70^{\circ}$. Les organismes échantillonnés à l'aide du filet Surber sont fixés sur le terrain avec une solution de formaldéhyde (5\%).

\section{4 - RÉSULTATS ET DISCUSSIONS}

\subsection{Physico-chimie}

Le tableau 2 présente les résultats concernant les paramètres mesurés.

Tableau 2 Valeurs du $\mathrm{pH}$, de l'aluminium total et de la conductivité pour chaque station.

Table $2 \quad \mathrm{pH}$, conductivity and total aluminium concentrations for each sampling site.

\begin{tabular}{|c|c|c|c|c|c|c|c|c|c|}
\hline \multirow{2}{*}{ Cours d'eau } & \multirow{2}{*}{$\mathbf{N}^{\mathbf{O}}$} & \multicolumn{4}{|c|}{ PH } & \multicolumn{2}{|c|}{ Auminlum } & \multicolumn{2}{|c|}{ Conductlvité } \\
\hline & & Minimum & Mayenne & Maximum & $\pi$ & $\begin{array}{c}\text { Moyenne } \\
\mu \mathrm{g} /\end{array}$ & $\mathbf{n}$ & $\begin{array}{l}\text { Moyenne } \\
\mu S / \mathrm{cm}\end{array}$ & $\pi$ \\
\hline Rau Saint Nicolas & 1 & 6,45 & 6,98 & 7,00 & 3 & 78 & 3 & 54,0 & 3 \\
\hline Thur & 2 & 6,50 & 6,89 & 7,05 & 5 & 49 & 3 & 45,0 & 3 \\
\hline Vologne & 3 & 6.54 & 6.91 & 7,04 & 4 & 60 & 3 & 69,0 & 3 \\
\hline Meurthe & 4 & 6,50 & 6,84 & 7,10 & 4 & 57 & 4 & 56,0 & 4 \\
\hline Bouchiot & 5 & 6,48 & 6,81 & 7,15 & 2 & 54 & 2 & 88,0 & 2 \\
\hline Rau des Bans & 6 & 6,52 & 6,80 & 7,26 & 10 & 51 & 4 & 55,0 & 4 \\
\hline Goutte du Tihay & 7 & 6,29 & 6,83 & 7,40 & 30 & 74 & 5 & 36,0 & 5 \\
\hline Rau des Echarges & 8 & 5,01 & 5,62 & 6,58 & 29 & 221 & 5 & 21,5 & $\mathbf{5}$ \\
\hline Rau du Grand Ventron & 9 & 4,62 & 5,18 & 6,02 & 29 & 236 & 5 & 19,5 & 5 \\
\hline Rouge-Rupt $673 \mathrm{~m}$ & 10 & 4,70 & 5,11 & 5,87 & 30 & 296 & 5 & 19,5 & 5 \\
\hline Gourte des Blancs Murgers & 11 & 4,47 & 5,01 & 5,89 & 27 & 256 & 5 & 17,0 & $\mathbf{5}$ \\
\hline Wassongoutte & 12 & 4,41 & 4,99 & 5,65 & 28 & 337 & 5 & 20,0 & 5 \\
\hline Goutte de la Grande Basse & 13 & 4,50 & 4,93 & 5,48 & 19 & 255 & 5 & 20,5 & 5 \\
\hline Rouge-Rupt $845 \mathrm{~m}$ & 14 & 4,30 & 4,84 & 5,29 & 18 & 301 & 5 & 19,0 & 5 \\
\hline Goutte du Pourri Faing & 15 & 4,10 & 4,75 & 5,40 & 28 & 371 & 5 & 20,5 & 5 \\
\hline Rouge-Rupt $1020 \mathrm{~m}$ & 16 & 4,13 & 4,67 & 5,07 & 28 & 387 & 5 & 20,5 & 5 \\
\hline
\end{tabular}


pH : Sept des quatorze cours d'eau étudiés (stations $8,9,10,11,12,13$, 14,15 et 16) sont caractérisés par des valeurs moyennes de $\mathrm{pH}$ variant entre 4,67 et 5,62 , avec un minimum de 4,10 à la station 15 et un maximum de 6,58 à la station 8 . Ces deux valeurs extrêmes correspondant respectivement à un événement pluvio-neigeux et à des conditions d'étiage accusé.

Les sept autres stations $(1,2,3,4,5,6$ et 7) montrent des $\mathrm{pH}$ moyens compris entre 6,8 et 7,0 , valeurs supérieures à celles des stations précédentes de 1,2 à 2,3 unités.

Aluminium total : Les sept ruisseaux acides présentent également les plus fortes concentrations moyennes en aluminium total. Toutes ces valeurs sont supérieures à $220 \mu \mathrm{g} / \mathrm{l}$. Rappelons ici que la valeur guide fixée par la CEE est de $50 \mu \mathrm{g} / \mathrm{l}$ et la norme maximale admise pour les eaux de consommation de $200 \mu \mathrm{g} / \mathrm{l}$. A l'inverse, les concentrations aluminiques moyennes relevées aux stations 1 à 7 sont de 3 à 9 fois plus faibles et ne dépassent pas $78 \mu \mathrm{g} / \mathrm{l}$.

Conductivité : Les conductivités moyennes des sept cours d'eau acides varient de 17 à $21,5 \mu \mathrm{S} / \mathrm{cm}$ et s'avèrent plus de deux fois plus faibles que celles relevées dans les ruisseaux non acides (36 à $90 \mu \mathrm{S} / \mathrm{cm}$ ).

Le calcul des corrélations entre pH et aluminium total $(r=-0,97)$ et $\mathrm{pH}$ et conductivité $(r=0,85)$ montrent que ces trois paramètres sont très fortement liés.

Compte tenu des caractéristiques physico-chimiques, nous avons considéré pour la suite de l'étude, deux groupes de cours d'eau :

Groupe 1 : Stations $1,2,3,4,5,6$ et 7 . Rivières non acidifiées, typiques du massif vosgien.

Gourpe 2 : Stations $8,9,10,11,12,13,14,15$ et 16 . Rivières acidifiées, faiblement minéralisées et présentant des concentrations aluminiques élevées.

Les rivières du groupe 2 n'hébergent plus de population de truite (Salmo trutta fario) alors que la présence de cette dernière était connue avec certitude dans les années 1960.

\subsection{Macro-Invertébrés benthiques}

Les espèces récoltées dans chacun des deux groupes de cours d'eau, sont répertoriées dans le tableau 3.

Quatre vingt seize espèces d'Ephéméroptères, de Plécoptères et de Trichoptères ont été recensées. Cet inventaire est le premier concernant un ensemble homogène de stations du massif vosgien. Les données antérieures sont éparses, anciennes et souvent imprécises quant à la détermination des taxons et/ou la localisation (MCLACHLAN, 1884, 1885, 1886, 1887 ; LESTAGE, 1924 ; HUBAULT, 1927 ; NAVAS 1931 et MOSELY, 1934), exception faite d'une note d'AUBERT (1963). Seules 26 espèces parmi les 93 récoltées en milieu non acide, ont été retrouvées dans les ruisseaux dont le pH est inférieur à 5,6, ce qui représente une réduction de plus de $70 \%$ de la richesse spécifique des trois groupes étudiés. 


\section{Ephémèroptères}

Vingt espèces d'Ephémèroptères ont été reconnues (tableau 3.a). Certaines d'entre elles sont très largement dominantes et semblent caractériser les peuplements du rhithron vosgien. Ainsi dans nos récoltes, Baetis alpinus, B. rhodani et Epeorus sylvicola représentent près de deux tiers des individus. Si l'on ajoute les Rhithrogena du groupe semicolorata et les deux espèces d'Ephemerella, on totalise plus de $80 \%$ des individus.

Tableau $3 a$ Liste des espèces d'Ephémèroptères récoltés dans les ruisseaux acides et non acides.

Table 3a List of Ephemeroptera species collected in acidic and non acidic streams.

\begin{tabular}{|c|c|c|}
\hline & Cours d'eau acides & Cours d'eau non acides \\
\hline Epeorus sylvicola (PICTET, 1865) & & + \\
\hline Ecotyonurus venosus (FABRCIUS, 1775 ) & & + \\
\hline Electrogena lateralis (CURTIS, 1775) & & + \\
\hline Rhitrogerna gr hercinia & & + \\
\hline R. picteti (SOWA, 1971) & & + \\
\hline R. puytoraci (SOWA et DEGRANGE, 1987) & & + \\
\hline Rhithrogena sp3 semicolorata & & + \\
\hline Siphlonunus lacustris (EATON, 1870\}) & & + \\
\hline Amelatus inopinatus (EATON, 1887) & & + \\
\hline Baetis rhodani (PICTET, 1843) & & + \\
\hline B. alpinus (PICTET, 1843) & & + \\
\hline B. melanonyx (PICTET, 1843) & & + \\
\hline B. vernus (CURTIS, 1834) & +()$\left.^{*}\right)$ & + \\
\hline B. niger (LINNE, 176t) & & + \\
\hline B. muticus (LINNE, 1758) & & + \\
\hline Ephemerella ignita (PODA, 1761) & & + \\
\hline E. mucronata (BENGTSSON, 1909) & & + \\
\hline Habroleptoides confusa (SARTONI et JACOB, 1986) & & + \\
\hline Paraleptophlebia submarginata (STEPHENS, 1835) & & + \\
\hline Habrophlebia lauta (EATON, 1884) & & + \\
\hline
\end{tabular}

(") 3 larves.

Dans les stations considérées, la répartition des Ephémèroptères en fonction du $\mathrm{pH}$ est très tranchée :

- Dans les ruisseaux non acides, la faune d'Ephémères est variée et abondante. Les cours d'eau comme la Goutte du Tihay, le ruisseau des Bans ou la Meurthe hébergent une quinzaine d'espèces.

- Dans les ruisseaux acides, les Ephémères sont absentes, à l'exception de deux affluents du Rouge-Rupt (stations 8 et 9) qui semblent comporter une faune d'Ephémèroptères probablement relictuelle puisque trois larves de Beatis vernus ont été récoltées en juillet 1989, période d'étiage durant laquelle le pH était supérieur à 5,8. 
La sensibilité globale de l'ordre des Ephémèroptères à l'acidité a été mise en évidence par de nombreux auteurs ; citons en particulier, en Amérique du Nord : FIANCE (1978) ; HALL et al. (1980, 1982) ; PETERSON et al. (1985) ; ALLARD et MOREAU (1987) ; en Scandinavie : ENGBLOM et LINGBELL (1984), RADDUM et FEILLJJHEIM (1984, 1987) ; en Grande Bretagne : SUTCLIFFE et CARRICK (1973), HARRIMAN et MORRISON (1982), WILLOUGBY (1988); et en Allemagne Fédérale : MATTHIAS (1983).

Cependant, certains genres semblent supporter d'assez bas pH (HENDREY et WRIGHT, 1975 ; ENGBLOM et LINGBELL, 1984). Ainsi le genre Leptophlebia, inféodé aux milieux lénitique, serait le plus résistant ; nous l'avons nous-même constaté dans les tourbières acides des Vosges (données non publiées). Toutefois, il convient d'être prudent quant à l'interprétation donnée à l'absence de certaines espèces. L'absence dans les cours d'eau acides étudiés, d'Ameletus inopinatus qui est une espèce peu fréquente dans les Vosges, semble liée essentiellement à sa rareté et non pas à l'acidité puisque, selon ENGLOM et LINGBELL (1984), OTTO et SVENSSON (1983), sa limite de tolérance se situerait vers une valeur de $\mathrm{pH}$ de 5 .

\section{Plécoptères}

Trente-cinq espèces de Plécoptères ont été identifiées dans les cours d'eau non acidifiés, chaque espèce mentionnée étant présente au moins en deux exemplaires (tableau 3.b).

Par contre dans les cours d'eau acides, seulement 17 espèces ont été capturées ce qui correspond à une diminution de plus de $50 \%$ de la richesse spécifique de ce groupe faunistique dans ce type de milieu.

Les Perlodidae et Perlidae, deux familles qui réunissent la plupart des espèces du sous ordre des Sétipalpia ne sont pas trouvées dans les eaux acides alors que Perlodes microcephala, Isoperla oxylepis, Diura bicaudata et Perla marginata sont communes dans les autres cours d'eau. Excepté Siphonoperla torrentium qui est récoltée en grand nombre, les Sétipalpes apparaissent donc très sensibles au processus d'acidification. Ceci est à rapprocher du fait que ces Plécoptères sont considérés comme les plus polluo-sensibles des invertébrés d'eau courante.

A linverse, quel que soit le type de cours d'eau considéré, les cinq familles de Plécoptères Filipalpes connues des Vosges sont présentes. Toutefois, certaines espèces de Leuctridae (Leuctra hippopus, $L$. aurita et $L$. alpina) et de Nemouridae (Protonemura praecox, $P$. nimborum, $P$. intricata, $P$. nitida, Nemoura marginata, $N$. cambrica, $N$. mortoni, $N$. avicularis) n'ont pas été identifiées dans les ruisseaux acidifiés. Dans ces mêmes cours d'eau certaines espèces sont très abondantes et les adultes sont récoltés en grand nombre à différentes époques de l'année, essentiellement au printemps et en été. C'est le cas de Brachyptera seticornis en avril, de Leuctra nigra en mai, de Siphonoperla torrentium en juin, et de Leuctra cingulata en juillet. 
Tableau 3b Liste des espèces de Plécoptères récoltés dans les ruisseaux acides et non acides.

Table $3 b \quad$ List of Plecoptera species collected in acidic and non acidic streams.

\begin{tabular}{|c|c|c|}
\hline & Cours d'eau acides & Cours d'eau non acides \\
\hline \multicolumn{3}{|l|}{ Filipalpia } \\
\hline Leuctra prima (KEMPNY, 1899) & + & + \\
\hline L. hyppopus (KEMPNY, 1898) & & + \\
\hline L. inermis (KEMPNY, 1899) & + & + \\
\hline L. pseudosignifera (AUBERT, 1954) & + & + \\
\hline L. rauscheri (AUBERT, 1957) & + & + \\
\hline L. cingulata (KEMPNY, 1899) & + & + \\
\hline L aurita (NAVAS, 1919) & & + \\
\hline L alpina (KUHTREIBER, 1934) & & + \\
\hline L. nigra (OLIVIER, 1911) & + & + \\
\hline Protonemura praecox (MORTON, 1894) & & + \\
\hline P. meyeri (PICTET, 1842) & + & + \\
\hline P. fumosa (RIS, 1902) & + & + \\
\hline P. nimborum ( $R I S, 1902$ & & + \\
\hline P. intricata (RIS, 1902 & & + \\
\hline P. lateralis (PICTET, 1902) & + & + \\
\hline P. nitida (PICTET, 1902) & & + \\
\hline Nemoura marginata (PICTET, 1902) & & + \\
\hline N. cinerea (RETZIUS, 19783) & + & + \\
\hline N. Cambrica (STEPHENS, 1835) & & + \\
\hline N. mortoni (RIS, 1902) & & + \\
\hline N. avicularis (MORTON, 1894) & & + \\
\hline Nemoura sp & + & + \\
\hline AmphinemUra sulcicollis (STEPHENS, 1835) & + & + \\
\hline Nemurella picteti (KLAPALEK, 1909) & + & + \\
\hline Capnia vidua (KLAPALEK, 1904) & + & + \\
\hline Brachyptera seticornis (KLAPALEK, 1902) & + & + \\
\hline B. nisi (MORTON, 1896) & + & + \\
\hline Taeniopteryx hubaulti (AUBERT, 1946) & & + \\
\hline \multicolumn{3}{|l|}{ Setipalpia } \\
\hline Perlodes microcephala (PICTET, 1842) & & + \\
\hline Perlodes Sp & & + \\
\hline Isoperla oxylepis (DESPAX, 1936) & & + \\
\hline Isopenta sp & & + \\
\hline Diura bicaudata (LINNE, 1758) & & + \\
\hline Perla marginata (PANZER, 1799) & & + \\
\hline Siphonoperla torrentium (PICTET, 1842) & + & + \\
\hline
\end{tabular}

L'appauvrissement de la faune plécoptèrique que nous avons constaté dans les ruisseaux acidifiés a également été mis en évidence au Canada (MACKAY et KERSEY, 1985), en Grande-Bretagne (SUTCLIFFE et CARRICK, 1973), en Scandinavie (RADDUM et FJELLHEIM, 1984) et en République Fédérale d'Allemagne (ZIEMANN, 1975). HALL ef IDE (1987) montrent en comparant des relevés faunistiques réalisés sur les mêmes cours d'eau d'Ontario en 1937 et 1985, la disparition de certains taxa dans les rivières dont le pH a baissé, en particulier la disparition de Plécoptères Perlidae et Perlodidae. MACKAY et KERSEY (1985) mentionnent des observations similaires. Les espèces apparte- 
nant à ces deux familles semblent donc particulièrement sensibles aux conditions acides du milieu.

L'absence d'espèces telle que Leuctra hippopus dans nos cours d'eau acidifiés, ne semble pas liée directement à l'acidité, car SUTCLIFFE et CARRICK (1973), HARRIMAN et MORRISON, (1982) et RADOUM et FJELLHEIM (1984) constatent sa présence dans des rivières acides.

\section{Trichoptères}

L'analyse des récoltes d'imagos, de nymphes et de larves dans certains cas, met en évidence sur l'ensemble des stations étudiées, 41 espèces de - Trichoptères parmi lesquelles trois espèces n'ont été prélevées que dans des cours d'eau acides (tableau 3.c). Il apparaît que seulement $21 \%$ des espèces présentes dans les ruisseaux non acides sont retrouvées dans les eaux dont le $\mathrm{pH}$ est inférieur à 5,6.

Les résultats montrent que les espèces résistantes aux conditions acides du milieu appartiennent essentiellement à trois familles : les Rhyacophilidae (Rhyacophila obliterata, R. philopotamoides centralis et R. praemorsa), les Polycentropodidae (Plectrocnemia sp et $P$. conspersa) et les Limnephilidae (Drusus discolor, D. annulatus, Chaetopterygopsis maclachlani, Chaetopteryx villosa et Anitella sp).

A l'inverse des Hydropsychidae (Hydropsyche sp), les Philopotamidae (Philopotamus ludificatus, P. montanus et Wormaldia $s p$ ) et les Glossoso matidae (Glossosoma conformis et Agapetus sp), qui représentent l'essentiel des récoltes dans les ruisseaux non acides, disparaissent complètement des cours d'eau dont le pH est inférieur à 5,6.

A côté de cette réponse nette, on remarque qu'une espèce, Odontocerum albicorne, n'a été trouvée en milieu acide (Station 10) que sous forme de jeunes stades larvaires en août et septembre.

L'acido-tolérance des Limnephilidae, Polycentropodidae et Rhyacophilidae a été rapportée par SUTCLIFFE et CARRICK (1973), HARRIMAN et MORRISON (1982), DIAMOND et al. (1987) dans des études portant sur des cours d'eau de Grande-Bretagne. HAVAS et HUTCHINSON (1982) mentionnent au Canada la tolérance de Limnephilidae pour des valeurs de pH inférieures à 4,0 alors que SIMPSON et al. (1985) constatent la présence de Rhyacophilidae dans des cours d'eau acides des Etats-Unis. En Scandinavie, ENGBLOM et LINGBELL (1984), RADDUM et FJELLHEIM (1984) remarquent la disparition de Philopotamus montanus quand le $\mathrm{pH}$ descend en-dessous de 6,0. Par contre en milieu lénitique au Pays Bas, LEUVEN et al. (1987), trouvent de nombreux taxa dans des eaux acides.

La sensibilité des organismes vis-à-vis des conditions rencontrées dans les eaux acides se traduit différemment d'un groupe à l'autre et l'on peut distinguer 3 niveaux de réponses:

- Extrême sensibilité des Ephémèroptères rhéophiles, à l'exception d'espèces appartenant à la famille des Leptophlebiidae, et des Plécoptères Sétipalpes. 
- Grande sensibilité des Trichoptères, mais certaines espèces réparties au sein de plusieurs familles semblent bien s'accomoder des conditions acides.

- Tolérance des Plécoptères Filipalpes qui constituent le groupe dominant dans les peuplements étudiés.

Tableau 3c Liste des espèces de Trichoptères récoltés dans les ruisseaux acides et non acides.

Table 3c List of Trichoptera species collected in acidic and non acidic streams.

\begin{tabular}{|c|c|c|}
\hline & Cours d'eau acides & Cours d'eau nan acides \\
\hline Rhyacophila aquatinica (MCLACHALN, 1879) & & + \\
\hline R. tristis (PICTET, 1834) & & + \\
\hline R. dorsalis (CURTIS, 1834) & & + \\
\hline R. evoluta (MCLACHLAN, 1879) & & + \\
\hline R. fasciata (HAGEN, SCHMID, 1970) & & + \\
\hline R. obliterata (McLACHLAN, 1863) & + & + \\
\hline R. philopotamoides centralis (SCHMID, 1970) & + & + \\
\hline R. praemosa (MCLACHLAN, 1879) & + & + \\
\hline Glossosoma conformis (NEBOISS, 1963) & & + \\
\hline Agapetus sp & & + \\
\hline Ptilocolepus granulatus (PICTET, 1834) & & + \\
\hline Philopotamus Iudificatus (McLACHLAN, 1878) & & + \\
\hline P. montanus (OONOVAN, 1813) & & + \\
\hline Wormaldia spi 1 & & + \\
\hline Womaldia sp2 & & + \\
\hline Hydropsyche sp1 & & + \\
\hline Hydropsyche sp2 & & + \\
\hline Plectrocnemia $s p$ & + & \\
\hline Psychomia pusilla (FABRICIUS, 1781) & & + \\
\hline Lype sp & & + \\
\hline Tinodes rostocki (McLACHLAN, 1878) & & + \\
\hline Micrasema bongutum (McLACHLAN, 1876) & & + \\
\hline M. minimum (MCLACHLAN, 1876) & & + \\
\hline Apatenia sp & & + \\
\hline Drusus annulatus (STEPHENS, 1837) & + & + \\
\hline D. discolor (RAMBUR, 1842) & + & + \\
\hline Anomalopteryx chauviniana (STEIN, 1874) & & + \\
\hline Limnephilus vittatus (FABRICIUS, 1798) & & + \\
\hline Encicyla pusilla (BURMEISTER, 1839) & & + \\
\hline Chaetopteryoopsis mactachlani (STEIN, 1874) & + & \\
\hline Chaetoperyx villosa (FABRICIUS, 1798) & + & \\
\hline Anitella Sp & + & \\
\hline Crunoecia irrorata (CURTIS, 1834) & & + \\
\hline Adicella reducta (MCLACHLAN, 1865) & & + \\
\hline Sericostoma personatum (KIRBY et SPENCE, 1826) & & + \\
\hline Odontocerum albicorne (SCOPOLI, 1763) & + & + \\
\hline
\end{tabular}


Dans le cas des cours d'eau, des données physico-chimiques ponctuelles, qui traduisent la qualité du milieu à un moment donné, ne permettent pas toujours la mise en évidence de l'acidification, notamment en dehors de périodes de stress acides et lors de conditions d'étiage prononcé. Par contre, les invertébrés benthiques intégrent les conditions du milieu tout au long de lannée, de sorte que leur réponse est la résultante des situations présentes mais aussi passées. Leur utilisation comme bio-indicateurs d'acidification semble donc s'imposer.

Cependant, pour être un bon bio-indicateur, un taxon (espèce, genre ou famille) doit satisfaire à plusieurs critères :

- taxon (espèce, genre, famille) à large répartition spatiale et temporelle, l'année,

- taxon (espèce, genre, famille) relativement abondant et présent toute

- taxon très sensible au facteur étudié,

- taxon d'identification aisée ou assez aisée à tous les stades du développement.

A titre d'exemples dans nos cours d'eau, Epeorus sylvicola, Perlodes microcephala, Hydropsyche, Philopotamus semblent présenter ces qualités.

II apparaît évident que la seule absence d'une espèce (genre ou famille) ne peut être prise en compte sans considérer les autres taxa.

Toutefois, it convient d'étendre les recherches à un nombre plus élevé de stations et de cours d'eau. Ainsi, une connaissance plus complète des peuplements de macro-invertébrés en relation avec une gamme plus variée de situations permettra de préciser l'écologie des différents taxons et leur réponses au stress acides et de dégager parmi eux des bio-indicateur pertinents.

\section{REMERCIEMENTS}

Ce travail a été effectué grâce au support financier du Ministère de l'Environnement (subvention $n^{\circ} 89020$ ).

Les auteurs remercient vivement MM. GIUDICELLI, SARTORI et THOMAS pour leur soutient quant à la détermination des espèces. Nous remercions également MM. G. GEHIN et M. GEHIN pour l'aide précieuse qu'ils nous ont apportée sur le terrain. 


\section{RÉFÉRENCES BIBLIOGRAPHIQUES}

ALLARD M., MOREAU G., 1987. Effects of experimental acidification on lotic macroinvertebrates community. Hydrobiologia, 144, $37-49$.

AUBERT J., 1963. Les plécopteres des Vosges. In : Le Honeck. Aspects physiques. biologiques et humains. Association philo. matique d'Alsace et de Lorraine. Ed. Stras bourg, 187-291.

BOURRIE G., 1976. Acquisition de la composition chimique des eaux en climat tempéré. Application aux granites des Vosges et de la Margeride, Thèse doctorat, Strasbourg, $214 p$.

BRINKHURST R.O., 1966. Detection and assessment of water pollution using Oligochaete worms. Parts 1 et 2. Water and $S e$ wage Works, Oct.-Nov., 8 p.

CAIRNS J., 1974. Indicator species vs. The concept of community structure as a index of pollution, Wat. Res. Bull., 10, 338-347.

COLIN J.L., RENARD D., LESCOAT V., JAF. FREZO J.L., GROS J.M., STRAUSS B., 1989. Relationship between rain and snow acidity. Atmos. Environ., 23, 1487-1498.

DIAMOND M., CRAWSHAW D.H., PRIGG R.F., CRAGG-HINE S., 1987. Streamwater chemistry and its influence on the distribution and abundance of aquatic inventebrates and fish in upland streams in Northwest England. In : Acid Rain. Scientific and Technical Advances. R. Perry Ed., Londres, $321 \mathrm{p}$.

ENGBLOM E., LINGDELL P.E., 1984. The mapping of short-term acidification with the help of biological pH indicators. Institute of freshwater Research, Drottingholm, repont 61, 60-68.

FIANCE S.B., 1978. Effects of pH on the biology and distribution of Ephemerelle funerais (Ephemeroptera). Okos, 31, 332-335.

GAUFIN A.R., TARZWELL C.P., 1952. Aquatic Invertebrates as Indicators Of Stream Pollu. tion. Public Health Reports, 67, 57-64.

GUEROLD F., PIHAN J.C., 1989. L'acidification des torrents vosgiens. Mise en évidence. Impact sur les populations de marcroinvertébrés. Revue des sciences de leau, 2, 621-640.
HALL R.J., LIKENS G.E., FIANCE S.B., HENDREY G.R., 1980. Experimental acidifica. tion of stream in the Hubbard Brook experimental forest, New Hampshire. Ecology, 61, 976-989.

HALL R.J., PRATT J.M., LIKENS G.E., 1982. Effects of experimental acidification on macroinvertebrate dritt diversity in a mountain stream. Water, Air and Soil Pollution, 18, 273-287.

HALL R.J., IDE F.P., 1987. Evidence of Acidification Effects on Stream insect Communities in Central Ontario Beetween 1937 and 1985. Can J. Fish. Aquat. Sci., 44, 16521657.

HARRIMAN R., MORRISON B.R.S., 1982. Ecology of streams draining forested and non forested catchments in area of central Scotland subject to acid precipitation. Hydrobiologia, 88, 251-263.

HAVAS K.E., HUTCHINSON T.C., 1982. Aquatic invertebrates from the smoking hills : effect of $\mathrm{pH}$ and metals on mortality. Can. J. Fish. Aquat. Sci., 39, 890-903.

HENDREY G.R., WRIGHT R.F., 1976. Acid precipitation in Norway. Effects on aquatic fauna. Ent. Journal Great Lakes Res., 2, 132-207.

HENRIKSEN A., 1979. A simple approach for identifying and measuring acidification of freshwater. Nature, 278, 542-545.

HUBAULT E., 1927. Contribution à l'étude des invertébrés torrenticoles. Bull. Biol. Fr. Belg., 9, 126-135.

LEIVESTAD H., MUNIZ I.P., 1976. Fishkill at low $\mathrm{pH}$ in a norwegian river. Nature, 259, 391-392.

LEUVEN R.S., HEMELRIJK J.A., VAN DER VELDE G., 1987. The distribution of Trichoptera in dutch soft water differing in pH. In : Proc. of the fith internat. symp. on Trichoptera. Lyon France. Par Junk Eds, Den Haag, 359-365.

LESTAGE J.A., 1924. Larves aquatiques d'insectes récoltées par MM. Cuénot et Rémy aux environs de Nancy (Meurthe et Moselle). Bull. Soc. Ent. France, 124, 123124. 
MACKAY R.J., KERSEY K.E., 1985. A preliminary study of aquatic insect communities and leaf decomposition in acid streams near Dorset, Ontario, Hydrobiologia, 122, 3-11.

MATTHIAS U., 1983. Der EinfluB des Versauerung auf die Zusammensetzung von Bergbachbiolzonosen. Schitzer Produktions biologische Studien (54). Arch. Hydrobiol., 65, 407-483.

MCLACHLAN R., 1884. Recherches névroptèrologiques dans les Vosges. Rev. Entomol., 3, 9-20.

MCLACHLAN R., 1885. Notes additionnelles sur les Névroptères des Vosges. Ibid., 4, $1-4$.

MCLACHLAN R., 1886. Notes additionnelles sur les Névroptères des Vosges (suite). bid., 5, 123-124.

MCLACHLAN R., 1887. Notes additionnelles sur les Névroptères des Vosges (suite). bid., 6, 57-58.

MOSELY M.E., 1934. A collecting trip in the Vosges and Bas-Rhin districts of France : Trichoptera, Plecoptera and Neuroptera. Entomologist, 67, 87-89.

NAVAS L., 1931. Insectos Neuropteros y atines de la Lorena (Francia). Broteria zoologica, 27, 137-144.

NORWEGIAN INSTITUTE FOR WATER RESEARCH, 1987. Convention on transboundary air pollution. (International cooperative programme on assessment and monitoring of acidification in rivers and lakes), Manuel for Chemical and Biological monitaring, Osto, $22 \mathrm{p}$.

ODEN S., 1976. The acidity problem. An outline of concepts. Water, Air, Soil pollution, 6, 137-166.

RETERSON R.H., GORDON D.J., JOHNSTON D.J., 1985. Distribution of maytly nymphs (Insecta ; Ephemeroptera) in some streams of Eastern pH. Can. Fields Naturalist, 99, 490-493.

PITTELKA L.F., RAYNAL D.J., 1989. Forest decline and acidic precipitation. Ecology, $70,2-10$.

RADDUM G.G., FJELLHEIM A., 1987. Effects of $\mathrm{pH}$ and aluminium on mortality, drift and moulting of the maytly Baetis rhodani. Annis Soc. r. Zool. Belg., 117, 77-88.
REHFUESS K.E., 1989. Acidic deposition - Extent and impact on forest soils, nutrition growth and disease phenomena in central Europe : A review. Water, Air and Soil Pollution, 48, 1-20.

SCHOFIELD C.L., 1976. Acid precipitation : Effects on fish. Acid precipitation : Effects on fish. Ambio, 5, 1-15.

SIMPSON K.W., BODE R.W., COLQUHOUN J.R., 1985. The macroinvertebrate fauna of an acid-stressed headwater stream system in the Adirondack Mountains, New York, Freshwat. Biol, 15, 671-681.

SCOTT W., 1989. Acid Rain : What We Know, What We Did, What We Will Do. Arch. Environ. Contam. Toxicol., 18, 75-82.

SUTCLIFFE D.W., CARRICK T.R., 1973. Studies on mountains streams in the English Lake District : $\mathrm{pH}$, calcium and the distribution of invertebrates in the river Duddon. Freshwat. Biol., 3, 437-462.

VAN COILLIE R., BROUARD D., LACHANCE M., VIGNEAULT Y., 1984. Possibilités écotoxicologiques des précipitations acides pour le saumon dans quatre rivieres a la cote nord du fleuve Saint-Laurent. Annis limnol., 20, 215-227.

VERNEAUX J. 1984. Méthodes biologiques et problèmes de la détermination des qualités des eaux courantes. Bull. Ecol., 15, 47-55.

WILLOUGHBY L.G., 1988. The distribution of Baetis muticus and Baetis rhodani (insecta, Ephemeroptera) with special emphasis on acid water background. Int. Rev. ges. Hydrobiol., 73, 259-273.

WOODIWISS F.S., 1964. A biological system of stream classification used by the Trend River Board. Chemistry and Industry, 11, 443-447.

WRIGHT J.F., ROSS D., ARMITAGE P.D., FURSE M.T., 1984. A preliminary classification of running water sites in Great Britain based on macro-invertebrate species and the prediction of community type using environmental data. Freshwat. Biol., 14, 221-256.

ZIEMANN H., 1975. Über den EinfluB der Wasserstoffionenkonzentration und des HydrogenKarbonatgehaltes auf die Ausbildung von Berbachbiozönosen. Int. Rev. ges. Hydrobiol., 60, 523-55 